\title{
Irrational use of antibiotics with representation of antimicrobial resistance patterns in Sudan: a narrative review
}

\author{
Noun Eltayeb Ahmed Abdulgader ${ }^{*}$, Rawan Khidir Abdel Galil², Shahd Yahaya Mohamed Nour \\ Serag Elnour ${ }^{2}$, Tabark Faisal Fadlallah Elhusain², Tagwa Mahmoud Ahmed Osman², Maymana Nadir \\ Abdelwahab Elawad ${ }^{3}$, Islam Mogbil Shaikh Idris Mirghani ${ }^{4}$, Ihab B Abdalrahman ${ }^{5,6}$, Sahar G. Elbager ${ }^{7}$
}

\section{Abstract}

\section{Background}

Increasing bacterial resistance to antibiotics is a growing menace, mainly caused by the rapid genetic modification of bacterial strains and new alternations in behavior favoring their survival. There is no doubt that the irrational use of antibiotics is one of the factors contributing to the rise of this problem, whether that be in hospitals or at a community level. Although the extent of this influence is yet to be learned, it is definite that this is of great impact on the endemic disease patterns in developing areas specifically and on an expanding global issue generally.

\section{Purpose and scope}

This paper will provide a narrative review of relevant previous publications of antibiotic misuse to portray a clearer picture of its causes and consequences in Sudan.

\section{Methodology}

The PICO method was used by which evidence-based research websites were scanned for key words. Results were assessed for relevance and then critically appraised. All papers included were summarized and presented in a narrative review format.

\section{Results}

From a total of 9 research papers from Pub Med, Scopus, Cochrane and Google Scholar search engines, 7 were selected, presented, and discussed.

\section{Conclusion}

Given the facts of high bacterial resistance that has emerged worldwide catastrophically, the implementation of a meticulous surveillance system designed to restrict the irrational use of antibiotics by the public and health sectors alike with adjunct educational and training programs relevant to the regional epidemiology and economy will massively contribute to a lower resistance rate due to antibiotic misuse.

${ }^{1}$ IIntensive care unit (ICU) Department, Ribat University Hospital,

Khartoum, Sudan.

${ }^{2}$ School of Medicine, Ahfad University for Women (AUW),

Khartoum, Sudan.

${ }^{3}$ School of Medicine, University of Medical Sciences and Technology,

Khartoum, Sudan

“Obstetrics and Gynecology Department, Dream Specialized Hospital,

Khartoum, Sudan.

${ }^{5}$ Acute Care Medicine, Soba University Hospital, Khartoum, Sudan.

${ }^{6}$ Associate Professor of Medicine, University of Khartoum, Khartoum,

Sudan.

${ }^{7}$ Department of hematology, Faculty of Medical laboratory sciences,

University of Medical Siences and Technology (UMST), Khartoum,

Sudan.

\section{Introduction}

As the world evolves through groundbreaking innovations and as technology development is on the rise, there is no denying that the discovery of antibiotic drugs in the $20^{\text {th }}$ century served a pivotal turn towards revolutionary breakthroughs in health care (1). It played a major role in the sustainability of life as we know it by minimizing threats to health.

Antibiotics have a wide range of uses; from treating serious infections, to preventing surgical wound infections, and as a prophylaxis for immunocompromised individuals (2). Inappropriate uses include multiple drug prescriptions (polypharmacy), over prescribing and over use of antibiotics, and irrational use of antimicrobials for infections that are viral in nature (3). Self-medication is a leading concern of one of the many irrational uses of antibiotics (4). This phenomenon is practiced in developing countries as well as developed countries alike. The dilemma of self medication in developing countries, such as Sudan, is brought about due to various issues such as limited purchasing power and insufficient knowledge about antibiotics. It is the choice of many due to multiple factors, however, a key reason is the patient's previous trial and knowledge of the efficacy of the 
treatment (4). The lenient behavior of community pharmacies in regards to dispensing antibiotics together with the expendable prescribing of antibiotics by physicians' results in unrestrained and excessive use that, in turn, raises the resistance of the human pathogens (4). Collective antibiotics consumption worldwide, essentially in developing countries, maximizes the antimicrobial resistance of the pathogen as the choices of effective antibiotics that are readily used to treat serious infections are narrowing (2). It is estimated that in some developing countries, $20.0 \%$ of antibiotics are used in hospitals and health care facilities while $80.0 \%$ are used in the community via prescription by a health care provider or an over the counter purchase (2). In Spain for example, outpatient prescriptions accounting for antibiotics alone are an alarming $92.0 \%$ whereas at an international scale it is a whopping $80.0 \%$ (5). An alarming approximate two-thirds of all oral antibiotics are dispensed without a prescription for treating various infections (4).

Tuberculosis (TB), caused by Mycobacterium tuberculosis, is considered a global health concern due to its growing resistance for anti-tuberculosis drugs. This adds to the burden of the disease with Sudan accounting for $14.6 \%$ of the total burden, ranking it as one of the high prevalence countries for TB (6). Moreover, there is a distressingly high rate of antibiotics prescribed for upper respiratory tract infections (URTIs) even though the majority is viral in nature, further encouraging the notion that antibiotics are effective for any URTIs $(4,15)$. Among the other common infections in Sudan necessitating antibiotic prescriptions are urinary tract infections and gastrointestinal infections, further amplifying the risk of microbial resistance to antibiotics (7).

Surgical site infections are also a leading concern to which the effectiveness of prophylactic antibiotics has been established (8). However, the growing use of prophylactic surgical antibiotics has been associated with resistance. Hence, precise selection of the appropriate antibiotic according to the anticipated bacteria at the surgical site as well as accurate timing of the dose and duration are critical. A single dose with a long enough half life to sustain activity throughout the procedure should suffice (8).

In our modern day and age, the decline in development of new generations of antibiotics alongside the inappropriate use of the currently available drugs brings about selective pressure on susceptible bacteria while favoring the survival of the resistant strains, some of which develop resistance to multiple antibiotics (9). Longer periods of infectivity, due to antibiotic misuse, increase the likelihood that more of the general population will contract the more resistant strains (4). Monitoring antimicrobial susceptibility profiles as well as regulating the dispensing of antimicrobials are some of the tools that could help prevent the spread of antibiotic resistance (6).

Along with the knowledge and surveillance system gaps, it is a given fact that antimicrobial resistance has put on an economic burden especially on sub-Saharan African countries, including Sudan, whose healthcare system is already overwhelmed (10-12). Unsurprisingly, results of extended-spec- trum beta-lactamase (ESBL) phenotypic activity has been found in some of the specific gram negative bacteria (10).

Taking irrational use of antibiotics from a different perspective, the magnitude of using it as a growth factor in the agricultural field in addition to any action that exposes antibiotics to the environment is considered a key for antimicrobial resistance. Different types of bacteria existing in the environment will become exposed to different types of antibiotics used and will inevitably result in resistance $(13,14)$.

The objective of this study is to summarize and collate this narrative review using available evidence-based researches of antibiotic misuse in various aspects of healthcare in Sudan, with hopes of assisting healthcare professionals in combating the spread of antibiotic resistance and the threat it poses.

\section{Material and Methods}

On the 10th of July 2020, the review title was established and the review question was formulated using the PICO method; which is a method used in clinical evidence practice that helps in building a framework for the research question in form of acrimony. The letters of the word PICO stand for: P: problem, population or patient, I: Intervention, C: comparison or control, O: outcome. Literature search was conducted through (PubMed, Scopus, Cochrane and Google scholar) databases, screening relevant data for "irrational use of antibiotics and antimicrobial resistance in Sudan", in addition to the relative key words. Subsequently, a few published papers in Sudan written in English were found and the reference lists of those papers were searched for more related articles. Critical appraisal and assessment for all articles were performed by all authors equally in order to maintain the scope of the literature and a number of papers were excluded from this review after analysis. Eventually, the selected papers underwent a precise retrieving and summarization process to formulate a standardized narrative review.
$\mathbf{P}$ : people misusing or overusing antibiotics
I: irrational use of antibiotics
C: irrational and rational use of antibiotics
O: antibiotics resistance

\section{Results}

In order to find accessible published papers in Sudan, a broad search was performed and 9 full research articles were found, 2 of which were not specifically related to the literature; therefore, 7 related full English research articles were included in this review. The following key words were used in the search (irrational, misuse, antibiotics, antimicrobial, and resistance). Eventually, the selected papers were presented and discussed.

\section{Discussion}

At the time of writing, there were few published papers conducted on irrational use of antibiotics and antimicrobial resistance in Sudan (2). Nonetheless, the absence of an antibiotic stewardship program that is applied and activated in Sudan's 
healthcare system is an alarming concern in regards to the issue of antibiotics resistance.

\section{Prevalence of irrational use of antibiotics in different levels of the community}

Despite the few numbers of data published in Sudan, the results shown were surprising and indicate an upcoming crisis in Sudan. However, the irrational use of antibiotics is practiced by doctors, pharmacists, and community members as shown in the study done by (Sara H Oleim et al, 2019) in River Nile State (RNS) in Sudan (2). Accordingly, patterns of antibiotics misuse in this study showed that $92.0 \%$ of doctors are prescribing antibiotics without culture and sensitivity tests while less than $10.0 \%$ depend on culture and sensitivity tests. $64.0 \%$ prescribe broad spectrum antibiotics while only $8.0 \%$ prescribe narrow spectrum. More than $93.0 \%$ of pharmacists dispense antibiotics without prescriptions, in addition to an overwhelming $89.0 \%$ of the public self-medicating with antibiotics (2). Giving the reasons behind self-medication with antibiotics, the study reported that lack of knowledge, absence of antibiotics dispensing strategies and pharmaceutical marketing are the most common significant factors in general, but more specifically, $43.0 \%$ of doctors reported that the main reason was unsatisfactory laboratory results. Moreover, this study reported that $82.0 \%$ of the participants used antibiotics for cough and common cold, $43.0 \%$ of them stopped antibiotics the moment they felt well, and the most commonly used antibiotic was Azithromycin representing at $27.0 \%(2)$.

Interestingly, another study done in Sudan by (Elhada et al, 2014) presented some variations from the previous study, in which $41.0 \%$ of the public reported self-medication with antibiotics, $38.0 \%$ of them self-medicated with Amoxicillin and $27.4 \%$ used antibiotics for cough and common cold (4).

On the other hand, a study done by (Mboya E et al, 2018) in Tanzania showed $88.8 \%$ used antibiotics while $76.3 \%$ of the participants used antibiotics without prescription. Presenting different complains, the most commonly reported complaint for using antibiotics was $48.0 \%$ upper respiratory tract symptoms (URTS), with the flu and cough being dominant. Participants in this study who used amoxicillin were $25.5 \%$ while, in contrast to the study done in Sudan, here azithromycin represented $6.2 \%$ of them. Eventually, this study highlighted that the poor knowledge of the population played a significant role in the irrational use of antibiotics, which further indicates the danger that African countries are going to face in the upcoming years (16). Moreover, another study done in Nigeria by (Akinyandenu $\mathrm{O}$ et al, 2014) focused on one of the major causes of antimicrobial resistance, which is the non-prescription sale of antibiotics, targeting pharmacists' practice. This study reported high rates of irrational use of antibiotics despite the prescription-only medicines (POMs) law activated in Nigeria (17).

Additionally, another study done in Sweden by (Machowska A et al, 2019) reviewing the irrational use of antibiotics across Europe clarified that the irrational use of antibiotics as well as the attitude of the community and healthcare workers towards antibiotics use are unfortunately attributed to the antimicrobial resistance among Europeans. Regarding that, 93.0\% of Europeans obtained antibiotics with a doctor prescription while only $4.0 \%$ of them had it without prescription, which is considered very low in comparison with the study done in Sudan. Despite the health system restrictions for antibiotics dispensing, the antimicrobial resistance concomitant to the irrational use of antibiotics is considered high for many reasons, represented in two major domains (18).

Community reasons:

- Accessible antibiotic dispensing

- Lack of awareness

- Re-using leftover antibiotics (2.0\% of Europeans)

Healthcare providers' reasons:

- Pharmacists and prescribers knowledge, attitudes and beliefs

- Delay of diagnostic tests

- Patient-doctor interaction

- Lack of training and educational programs

- Pharmaceutical promotions

All those reasons are similar to the reasons responsible for the irrational use of antibiotics in Sudan, which indicate a serious problem at a worldwide scale (18).

\section{Irrational use of antibiotics among hospitalized pa- tients}

According to a study done by (Elfaki A, et al, 2009) highlighting the irrational use of antibiotics in hospitalized patients and that has been included in this review, antimicrobial resistance has become a global concern and particularly a life threatening issue in Sudan and studying it in different settings has become an imperative demand. However, in this study $58.5 \%$ of hospitalized patients have received antibiotics from their medical records and cephalosporin was found to be a $34.5 \%$ of all classes of dispensed antibiotics, whereas ceftriaxone represents $16 \%$ of all prescribed antibiotic drugs (3). In contrast, a study done by (Abdulah R, 2012) in Indonesia showed that 44.0\%-97.0\% of hospitalized patients were prescribed antibiotics (19). Surprisingly, the rates of inappropriate usage of antibiotics in hospitalized patients were challenging and even extended to developed countries such as the United States of America (USA), where the Centre of Disease Control (CDC) reported that $55.7 \%$ of hospitalized patients received antibiotics (20). However, a study done in Pakistan by (Saleem $Z$ et al, 2019) showed relatively similar results of our study, representing $53.7 \%$ of hospitalized patients in public sectors and $46.3 \%$ in private sectors along with cephalosporin being the most commonly prescribed class of antibiotics that represented $46.1 \%$ (20). Subsequently, those presented results indicate an alarming threat the community is unaware of.

\section{Irrational use of antibiotics as a prophylaxis in the obstetrics and gynecology department}

As part of the irrational use of antibiotics, a study was done in Sudan by (Elbur A et al, 2014) regarding the use of antibiotics as a surgical prophylaxis in obstetrics and gynecology wards 
given the high rates of surgical site infections. This study revealed $98.8 \%$ of admitted cases received antibiotics pre-operatively while $100.0 \%$ received antibiotics post-operatively for an average duration of 8 days. In addition, $92.6 \%$ was reported for cefuroxime use in this study pre-operatively with metronidazole being $99.0 \%$ post-operatively (8). In contrast, a study done in a Canadian hospital by (Achong $\mathrm{M}$ et al, 2015) showed that out of 76 patients admitted to the gynecological ward, 40 of them received prophylactic antibiotics, $77.5 \%$ were Cephalothin. This study conducted the antibiotic therapy assessment for different aspects including the choice of the antibiotics, the dosage and whether it is indicated or not, and that was considered strongly critical for such an assessment (21).

\section{Isolated pathogens resistant to specific antimicrobi- als}

Given the fact that the most commonly reported infections treated with antibiotics are the respiratory ones, a study done in Khartoum, Sudan by (Ahmed S et al, 2018) isolating respiratory samples taken from different levels of the respiratory tract according to antibiotic susceptibility testing ran in this study, gram negative bacteria were found to be $52.69 \%$ while $47.31 \%$ represent gram positive bacteria. Moreover, Klebseilla spp. and Mycobacterium tuberculosis were the most common organisms sharing the same percentage of $25.2 \%$. Additionally, the highest resistance of gram negative as well as gram positive was to penicillin, in which it represented $100.0 \%$ for gram negative and $88.0 \%$ for gram positive (6).

In concordance with this study, another one was done in Sudan by (Ahmed A et al, 2000) tracking bacterial isolate from urine and diarrhea specimens. Regarding urinary pathogens, Klebsiella pneumoniea (K. pneumoniea) represented 90.0\% resistance to ampicillin and Escherichia coli (E. coli) showed $75.0 \%$ resistance, while both presented low resistance to cephalexin where E. coli is $3.0 \%$ and Klebsiella spp. is $12.0 \%$. Subsequently, enteric pathogens showed $59.0 \%$ of Shigella flexneri resistant to amoxicillin and $90.0 \%$ of Shigella dysentriea resistant to ampicillin (7). Moreover, another study done by (Ibrahim A et al, 2014), with the samples collected from Sudan and Saudi Arabia found that $97.7 \%$ of E. coli isolate from Sudan was resistant to amoxicillin, similar to the isolate specimens of Saudi Arabia which represented 96.7\% (22).

Since antimicrobial resistance is considered a worldwide problem, results from a study done in the USA by (Walker G et al, 2019) seemed relatively similar to our studies, in which different isolate specimens from different geographical regions showed $88.0 \%$ resistant phenotype to Ampicillin while K. pneumonea has intrinsic resistance to it and $90.0 \%$ resistance to cefazolin. Generally, E. coli was found to be $91.0 \%$ phenotypic resistant and $K$. pneumonea was $93.0 \%$ across different antibiotics types (23). Tracking E. coli and $K$. pneumonea resistance rate, a study done by (Remschmidt $C$ et al, 2017) also showed a high rate of resistance to third-generation cephalosporin .

Together with previously mentioned microbial resistance, food-borne infections caused by campylobacters are needed to be stopped in Sudan. Herein, the previously mentioned study (7) illustrated that campylobacters are $100 \%$ resistant to Cotrimoxazole, in contrast to a study done in the USA by (Riso A et al, 2017) in which campylobacter showed $43.0 \%$ resistance to tetracycline (25).

In order to fight the antimicrobial resistance in Sudan, we should come up with the bigger picture and solve the problem radically. It's worth mentioning that Helicobacter pylori $(H$. pylori) accounts for some schoolchildren infections, where it represents $21.8 \%$ in a study done in Sudan by (Abbas M et al, 2018) (26), compared with a $54.8 \%-75.0 \%$ range of prevalence in East Africa, 45.7\% - 65.0\% in Tanzania (Jaka H et al, 2018) (27) and 79.3\% in Cameroon (Mabeku l et al, 2019) (28).

\section{Conclusion}

In conclusion, all the data emphasized that the leading causes of antimicrobial resistance in Sudan are multifactorial, being presented in different angles. The irrational use of antibiotics practiced by physicians, pharmacists and the public are causes of complexity in this issue. Additionally, lack of public awareness is a well-recognized cause playing a major role in the widespread of this crisis.

In order to combat and eradicate the roots of this problem, it is essential to build a restricted local stewardship program and implementing it according to the epidemiological data of potential pathogens and the available resources in Sudan. Accordingly, every training program should have a proposed strategy to control the increase in drug resistance and to prolong the usefulness of antibiotics. The irrational use of antibiotics is affecting the healthcare system as well as the economy worldwide, therefore, international collaboration and unity are needed to enhance and progress through the target.

\section{Conflict of interest and Acknowledgement}

Authors have declared that no competing interests exist. This work is dedicated to our families who were the key to our success along the way as well as to our colleagues Dr. Abdalrahman Hamza, Dr. Sahar Albagir and Mona Yahia Ibrahim Elghosain who guided us through this work; your efforts are highly appreciated. Lastly but not least, a special appreciation to Dr. Mohamed Nasr Elshiekh for his unlimited giving during this scientific research journey.

\section{Ethical Review and Approval}

Not applicable

\section{References}

1. Aminov R. A brief history of the antibiotic era: lessons learned and challenges for the future. 10.3389/ fmicb.2010.00134

2. Oliem S, Noor S, Bushara S, Ahmed M, Elmadhoun W. The Irrational Use of Antibiotics Among Doctors, Pharmacists and the Public in River Nile State, Sudan. Sudan Journal of Medical Sciences 2019;10.18502/sjms.v14i4.5909

3. Elfaki A. Assessment of Antibiotics prescription in Hos- 
pitalized Patients at Elobeid Hospital, Sudan. Sudan JMS 2009;4(3)

4. Elhada A, Eltayeb I, Mudawi M. PATTERN OF SELF-MEDICATION WITH ANTIBIOTICS IN KHARTOUM STATE, SUDAN. World Journal of Pharmaceutical Research 2014; 678-692

5. Sanchez M, Vallina-Victorero M, Bachiller M, Arbizu R, Llaneza E, Rozada S, et al. Variability in the community consumption of antibiotics: a problem in Europe, Spain and Asturias. Le Infezioni in Medicina, n 2019; 2, 134-140

6. Ahmed S, Abdelrahman S, Saad D, Osman I, Osman M, Khalil E. Etiological Trends and Patterns of Antimicrobial Resistance in Respiratory Infections. The Open Microbiology Journal 2018; 12, 34-40

7. Ahmed A, Osman H, Mansour A, Musa H, Ahmed A, Karrar Z, et al. ANTIMICROBIAL AGENT RESISTANCE IN BACTERIAL ISOLATES FROM PATIENTS WITH DIARRHEA AND URINARY TRACT INFECTION IN THE SUDAN. Am. J. Trop. Med. Hyg 2000; 259-263

8. Elbur A, Yousif M, El Sayed A, Abdel-Rahman M. Misuse of prophylactic antibiotics and prevalence of postoperative wound infection in obstetrics and gynecology department in a Sudanese hospital. Health 2014; 158-164

9. Bell B, Schellevis F, Stobberingh E, Goossens H, Pringle M. A systematic review and meta-analysis of the effects of antibiotic consumption on antibiotic resistance. BMC 2014; 1471-2334/14/13

10. Toy T, Pak G, Duk T, Campbell J, El Tayeb M, Kalckreuth $\mathrm{V}$, et al. Multicountry Distribution and Characterization of Extended-spectrum $\beta$-Lactamase-associated Gram-negative Bacteria From Bloodstream Infections in Sub-Saharan Africa. Clinical Infectious Diseases 2019; 69(S6):S449-58

11. Tadesse B, Ashley E, Ongarello S, Havumaki J, Wijegoonewardena M, González I, et al. Antimicrobial resistance in Africa: a systematic review. BMC Infectious Diseases 2017; 17:616

12. Mouiche M, Moffo F, Akoachere J, Okah-Nnane N, Mapiefou N, Ndze V, et al. Antimicrobial resistance from a one health perspective in Cameroon: a systematic review and meta-analysis. BMC Public Health 2019; 19:1135

13. Escudeiro P, Pothier J, Dionisio F, Nogueira T. Antibiotic resistance gene diversity and virulence gene diversity are correlated in human gut and environmental microbiomes. mSphere 2019; 4:e00135-19

14. Akova I, Baquero F, Carlet J, Cavaleri M, Coenen S, Cohen J, et al. The global threat of antimicrobial resistance: science for intervention. New Microbe and New Infect 2015; 6: $22-29$

15. Pearson M, Chandler C. Knowing antimicrobial resistance in practice: a multi-country qualitative study with human and animal healthcare professionals. GLOBAL HEALTH ACTION 2019;1599560

16. Mboya E, Sanga L, Ngocho J. Irrational use of antibiotics in the Moshi Municipality Northern Tanzania: a cross sectional study. Pan African Medical Journal 2018;31:165.
17. Akinyandenu $\mathrm{O}$, Akinyandenu A. Irrational use and non-prescription sale of antibiotics in Nigeria: A need for change. Journal of Scientific and Innovative Research 2014; 3 (2): 251-257

18. Machowska A, Lundborg C. Drivers of Irrational Use of Antibiotics in Europe. Int. J. Environ. Res. Public Health 2019;16, 27

19. Abdulah R. Antibiotic Abuse in Developing Countries. Pharmaceut Reg Affairs 2012; 1000e106

20. Saleem Z, Saeed H, Hassali M, Godman B, Asif U, Yousaf $\mathrm{M}$, et al. Pattern of inappropriate antibiotic use among hospitalized patients in Pakistan: a longitudinal surveillance and implications. Antimicrobial Resistance and Infection Control 2019; 8:188

21. Achong M, Hauser B, Krusky J. Rational and irrational use of antibiotics in a Canadian teaching hospital. CMA JOURNAL/FEBRUARY 2015; 1977/VOL. 116

22. Ibrahim M, Bilal N, Hamid M. Comparison of Phenotypic Characteristics and Antimicrobial Resistance Patterns of Clinical Escherichia coli Collected From Two Unrelated Geographical Areas. Global Journal of Health Science 2014; 1916-9736

23. Walker GT, Quan J, Higgins SG, Toraskar N, Chang W, Saeed A, et al. Predicting antibiotic resistance in Gramnegative bacilli from resistance genes. Antimicrob Agents Chemother 2019; 63:e02462-18.

24. Remschmidt C, Schneider S, Meyer E, Schroeren-Boersch B, Gastmeier P, Schwab F. Surveillance of antibiotic use and resistance in intensive care units (SARI) - a 15-year cohort study. Dtsch Arztebl Int 2017; 114: 858-65

25. Facciola A, Riso R, Avventuros E, Visalli G, Delia S, Lagana P. Campylobacter: from microbiology to prevention. J PREV MED HYG 2017; 58: E79-E92

26. Abbas M, Sharif F, Osman S, Osman A, El Sanousi S, Magzoub M, et al. Prevalence and Associated Symptoms of Helicobacter pylori Infection among Schoolchildren in Kassala State, East of Sudan. Hindawi Interdisciplinary Perspectives on Infectious Diseases 2018; 4325752

27. Jaka H, Rhee J, Östlundh L, Smart L, Peck R, Mueller A, et al. The magnitude of antibiotic resistance to Helicobacter pylori in Africa and identified mutations which confer resistance to antibiotics: systematic review and meta-analysis. BMC Infectious Diseases 2018; 18:193

28. Mabeku L, Bille B, Zemnou C, Nguefack L, Leundji F. Road spectrum resistance in Helicobacter pylori isolated from gastric biopsies of patients with dyspepsia in Cameroon and efflux-mediated multiresistance detection in MDR isolates. BMC Infectious Diseases 2019; 19:880 